\title{
3-S26-1
}

\section{Relationship between AGE scavenging and angiogenesis in endothelial cell}

\author{
Takashi Nishinaka $^{1}$, Omer Faruk Hatipoglu ${ }^{1}$, Hidenori Wake ${ }^{1},{\text { Shuji } \text { Mori }^{2} \text {, Masahiro Nishibori }}^{3}$, \\ Hideo Takahashi ${ }^{1}$
}

${ }^{1}$ Dept. Pharmacol., Facul. Med., Kindai Univ., ${ }^{2}$ Dept. Pharmcol., Sch. Pharmacy, Shujitsu Univ., ${ }^{3}$ Dept. Pharmacol., Sch. Med. Dent. Pharm. Sci., Okayama Univ.

Advanced glycation end products (AGEs) are biologically reactive compounds associated with diabetic complications and aging-related disorders. Exposure to AGEs in endothelial cells lead to excess angiogenesis, however, molecular mechanisms underlying AGE-elicited angiogenesis remain unclear. Many types of receptors, such as receptor for AGEs (RAGE), toll like receptor-4 and scavenger receptors, are expressed in endothelial cells and contribute to the AGE-elicited alteration of cell function. Scavenger receptors has been thought to play a significant role in the recognition and elimination of AGEs from the circulation. The involvement of scavenger receptors on the AGEelicited excess angiogenesis is still unknown. We found that three types of scavenger receptors (CD36, CD163 and lectin-like oxidized LDL receptor-1; LOX-1) contribute to the AGEs-induced enhancement of angiogenesis. The cocktails of neutralizing antibodies against CD36, CD163 and LOX-1 prevented tube formation but not AGE uptake. On the other hand, AGE-RAGE pathway is indirectly involvement in enhancement of tube formation mediated by upregulation of scavenger receptors on cell surface. Fucoidan, which is sulfated polysaccharide derived from brown algae and non-selective scavenger receptors inhibitor, suppressed AGE-elicited tube formation. Our findings propose that therapies drug for AGE-relating disorders need to have capacity inhibiting multiple scavenger receptor in endothelial cells. 